\title{
Modeling discrete twin lamellae in a microstructural framework
}

\author{
Marko Knezevic $^{\mathrm{a},}{ }^{*}$, Mark R. Daymond $^{\mathrm{b}}$, Irene J. Beyerlein ${ }^{\mathrm{c}}$ \\ a Department of Mechanical Engineering, University of New Hampshire, Durham, NH 03824, USA. \\ bDepartment of Mechanical and Materials Engineering, Queen's University, ON K7L3N6, Canada. \\ ${ }^{c}$ Theoretical Division, Los Alamos National Laboratory, Los Alamos, NM 87545, USA.
}

\begin{abstract}
The impact of deformation twins on the mechanical response of metals derives from the fact that they are microstructural inhomogeneities that create lattice reorientation and introduce local stress fields. Efforts have begun towards microstructural modeling of discrete twin domains within individual grains and the stress and strain fields that result from them. The aim of this viewpoint article is to review these endeavors, highlighting the insights gained by these studies, the advantages and disadvantages of the seemingly diverse approaches taken to date, and on this basis, offer our perspective on promising future steps.
\end{abstract}

Keywords: Texture; Mesostructure; Twinning; Dislocations; Crystal Plasticity

Twinning is one of the basic deformation mechanisms by which individual crystals accommodate mechanical strain [1,2]. Deformation twins are discrete domains that are believed to begin as atomic scale embryos that grow with applied strain to become grain-scale lamellae. Twin domains are crystallographically reoriented with respect to the parent grain, which introduces a boundary between parent and twin domain a 'twin boundary'. Formation of a twin shears the material by a characteristic amount, the twin shear, on the twin plane and in the twinning direction $[1,3,4]$.

Plastic deformation in polycrystalline metals is most often accommodated by plastic slip, but under certain circumstances can be carried by both twinning and slip. These conditions are specific to the material and twin mode, but generally twinning is reported to occur easily at high loading rates, low temperatures, and under particular states of strain $[1,5,6]$.

The macroscopically observed stress-strain response is radically different when twinning and slip together accommodate plastic deformation than when plastic slip acts alone. Figure 1 shows examples of the tension-compression asymmetry that can arise in materials that twin readily under ambient pressures and quasi-static rates. These metals possess a low symmetry crystal structure and are $\alpha$-titanium ( $\alpha$-Ti) [7], $\alpha$-zirconium $(\alpha-\mathrm{Zr})$ [8], a magnesium alloy (AZ31) [9], and $\alpha$-uranium $(\alpha-U)$ [10]; all results are obtained from sheet material with a strong texture. Due to the choice of applied load direction relative to the sheet texture and allowed deformation modes, in each of the tension cases, deformation was accommodated by slip, whereas in compression it was accommodated by slip and twinning. In the predominantly slip (tension) curves, the hardening rate reduces with applied strain, while in the twinning and slip

\footnotetext{
* Corresponding author at: University of New Hampshire, Department of Mechanical Engineering, 33 Academic Way, Kingsbury Hall, W119, Durham, New Hampshire 03824, United States. Tel.: 603862 5179; fax: 603862 1865.

E-mail address: marko.knezevic@unh.edu (M. Knezevic).
} 
(compression) curves, there are inflection points in the hardening rate, and over some periods the hardening rate increases with applied strain.

Fig. 1. Effect of deformation twinning on the compressive response of: (a) AZ31 [9], (b) $\alpha$-Ti [11], (c) $\alpha-\mathrm{Zr}$ [12], and (d) $\alpha-U$ [10]. Response in tension for all these metals exhibits characteristics of slip dominated deformation.

Arguably many factors could be responsible for the differences seen in the twinning responses but two are worth highlighting: the preferred slip systems and the morphology of the twin domains. For the three HCP crystal systems shown, in the Mg alloy (AZ31) the easiest slip system is basal slip, whereas in $\alpha$-Ti and $\alpha-\mathrm{Zr}$ it is prismatic slip [2, 13-15]. In $\alpha-\mathrm{U}$, which has an orthorhombic crystal structure, the easier slip system is floor slip [16-19]. Twin morphologies can be easily seen in EBSD maps, such as those in Fig. 2, which correspond to the compressed materials in Fig. 1. As shown, the twins manifest as discrete domains within a majority of grains. The crystal lattice within the twin domain has significantly reoriented from that of the parent grain and hence both twin volumes and twin boundaries can be easily exposed. Despite length scale differences relative to parent grain size, some key differences can be observed: in $\mathrm{Mg}$, the twins are few and thick; in $\mathrm{Zr}$ and $\mathrm{Ti}$, they are many and thinner compared to $\mathrm{Mg}$; in $\mathrm{U}$, the twins are numerous and very thin.

Such morphological details of the twin can locally affect which type of slip is favored and hence influence the macroscopic stress-strain response. The twin introduces a new crystallographic orientation into the grain and within this domain, slip systems can be preferred that are different from those in the parent. The directional boundaries introduced by twins can pose obstacles against slip motion and depending on the relative orientation of the slip mode and twin boundary, the barrier can be stronger for one slip system than another. A third way that twin morphology can take affect, which is neglected, is through the local stress states that are generated within the twin and surrounding matrix. The localized stress-strain fields produced by twins, in addition to creating localized hardening, influence local slip activity within the parent grain and twin itself, formation of additional twins, and twin expansion rates [20]. Knowledge of these local stresses and strains could help explain the probability of other twin-related processes, such as twin-twin transmission [21], secondary twinning, and de-twinning [22-24]. Furthermore, through the local stresses that they generate, twins are thought to play a role in recrystallization kinetics [25, 26], fatigue, [27], and ductile fracture [28].

Fig. 2. Inverse pole figure maps showing microstructure evolution in samples compressed along the in-plane rolling direction of sheets of (a) AZ31 to a true strain of 0.04 at $298 \mathrm{~K}$ [9], (b) $\alpha$-Ti to a true strain of 0.1 at $298 \mathrm{~K} \mathrm{[11],} \mathrm{(c)} \alpha-\mathrm{Zr}$ to a true strain of 0.08 at $76 \mathrm{~K}$ [12], and (d) $\alpha-\mathrm{U}$ to a

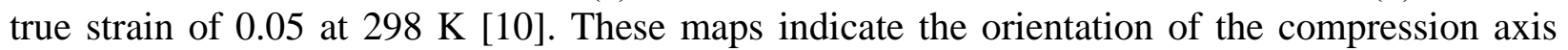
with respect to the crystal reference frame.

To date, we lack a constitutive model that accounts for the changing morphology of the twin, the hardening effect introduced by twin boundaries, and the activation of slip and twinning systems according to the local stresses produced by twins. Most constitutive models for metals that deform by combinations of slip and twinning utilize mean-field polycrystal models, such as Taylor-type [29, 30] and self-consistent [31] schemes. In these approaches, the neighborhood is homogenized and hence the grain boundaries and grain neighbors are not explicitly modeled. 
Within mean-field codes, four micromechanics approaches currently exist for introducing a reoriented twin phase: (i) predominant twin reorientation (PTR) method [32, 33], (ii) volume fraction transfer (VFT) scheme [33], (iii) total Lagrangian approach [34], and (iv) compositegrain method $[35,36]$. The twin phase replaces some fraction of the matrix phase and as the volume of the twin phase increases with strain, the volume of the matrix phase shrinks accordingly. With such sub-grain twinning models polycrystal models have been successful in demonstrating many key effects of twins on the macroscopic stress-strain response and texture evolution. However, such schemes assume homogeneous stress states in the matrix and twin phases, which can be expected to be very different from the actual highly localized stresses produced by twin domains, as have been reported experimentally using techniques such as farfield 3D-XRD [20, 37-39] and differential-aperture X-ray microscopy (DAXM) [40].

Localized stresses as a result of inhomogeneities, such as twins, can be calculated using spatially resolved mechanics models. To date three types have been used: phenomenological finite element (PFE) [41, 42], crystal plasticity fast Fourier transform (CPFFT) [43], and crystal plasticity finite element (CPFE) [44]. These techniques can calculate the spatially resolved mechanical fields within deformed microstructures, thereby overcoming the local stress state limitations of mean-field techniques. They account for grain-grain interactions and predict gradients in strains, orientations, and stresses across grains and grain boundaries. In the case of PFE, the material point is a continuum. In CPFFT and CPFE, the material point could be a single crystal or a Taylor or VPSC polycrystal [45-49]. Traditionally, however, deformation at each material point has been governed solely by elasticity and plasticity, and not twinning. Only recently have efforts begun towards modeling discrete twin lamellae in a microstructural framework using such techniques.

For many reasons, incorporating twinning at each material (integration or grid) point in these techniques is not as straightforward as slip. Unlike slip, twinning accommodates strain by creating an inhomogeneity. Also, understanding where a twin forms, how it expands and the relevant driving forces is still not well developed. Last, advancement has also been hampered by the lack of 3D microstructural representation codes [50], computational power [51], and efficient geometry and mesh manipulation tools.

One of the earliest studies to explicitly incorporate twins in an FE setting was carried out by Zhang et al. [41, 52]. The objective was to study the local stress distributions surrounding a twin in $\mathrm{Zr}$.

Fig. 3. A 2D FE model for twinning with a square inclusion represents a grain embedded in an isotropic surrounding medium. Von Mises stress contour before (a) and after (b) twinning under tension along the global $X$ direction [41].

Some results from PFE example [41] are shown in Fig 3. The twinned grain was modeled as a thin lamella chosen to correspond to a $(10 \overline{1} 2)[\overline{1011}]$ twin within a rectangular parallelepiped single crystal parent grain inclusion. The twin planes span the entire grain. The twin-parent ensemble was embedded in an isotropic, perfectly plastic homogeneous effective medium representing the polycrystal. The twin was an inhomogeneity possessing the elastic and plastic anisotropy associated with the orientation of the twin with respect to the matrix, and a single crystal yield surface was approximated for the grain. The ensemble was subjected to plane strain compression. The study highlighted for the first time the highly heterogeneous stress fields associated with an inhomogeneous twin domain, and suggested a stress driver for lenticular twin 
formation. Additionally, the study correlated the stress states that are energetically favorable for twinning with twin volume fraction and morphology, and the conditions under which multiple twins might be favored within a single grain as opposed to a single larger twin.

More recently, the CPFFT method was used to study distributions of local stress states around a twin in $\mathrm{Mg}$ [43] (Fig. 4). The model was a tri-crystal comprised of a central grain containing a twin, flanked by two identical neighboring grains. The method of solution is based on a Green's function technique and hence the CPFFT model needs to be discretized into a regular grid. The twin lamella was incepted into the central grain while the tricrystal was placed under an applied compression stress of $40 \mathrm{MPa}$. The twin was in the shape of a lamella defined by two parallel interfaces corresponding to a $(01 \overline{1} 2)[0 \overline{1} 11]$ tensile twin boundary. The twin connected the two ends of the grain, as in [40]. In this study, the thickness of the twin, or the number of voxels that spanned the twin, ranged from 7 to 13 , corresponding to $0.5 \%$ to $1 \%$ twin volume fraction in the parent. The preselected twin-domain voxels are reoriented such that the proper twin-parent orientation relationship is achieved. Then to every twin-domain voxel, the twin shear transformation is enforced. After the twin was incorporated, the authors studied the stress and strain fields that had been generated. For each voxel, both inside and outside the twin, the simulation used a crystal plasticity based elastic-visco-plastic constitutive law with no hardening in the CRSS for slip. The resulting distribution was predominantly controlled by plastic relaxation.

In order to gain insight into further twin growth, the authors of [43] studied the resolved shear stress contours corresponding to the twin variant created. They found the stress state to be highly non-uniform, as a result of the material's reaction of the localized shear imposed by the twin domain. In particular, the twin-resolved shear stress component was positive in the neighboring grain near the twin/grain boundary junction, negative along the twin boundary in the parent grain, and negative within the twin lamella (see Fig. 4, red line profile). The local negative twin-resolved shear stresses represent a backstress imposed by the surrounding crystals, suggesting that the twin transformation itself hinders subsequent expansion of the twin and more applied stress would be required to expand the twin further. The calculations also showed that the backstress was lower in the central part of the twin lamella, suggesting that if the applied stress were to increase the twin would expand in the middle (see Fig. 4, the blue line profile). They repeated simulations with the same parent/twin orientation but with different neighbor orientations. They concluded that other neighboring grain orientations produced the same qualitative results, with a high backstress in the twin lamella at the twin/grain boundary junction and a lower one at the center of the lamella.

Fig. 4. (a) Schematic of the tricrystal unit cell compressed in the 2-direction with a twin inside the central crystal and (b) the resolved shear stress profile along the twin-parent grain boundary [43].

Explicit twinning geometries have been studied in HCP materials within a CPFE framework $[44,53,54]$; as an example, we discuss a recent study on polycrystalline $\alpha-U$. There, the CPFE technique is modified to include discrete twin lamellae with their associated twin boundary, twin-matrix reorientation relationship, and shear transformation strain [44]. Polycrystalline $\alpha-U$ is highly elastically and plastically anisotropic and hence the authors implemented a crystal plasticity based constitutive law including elastic anisotropy and rate-dependent plasticity occurring on possibly four distinct slip modes and two deformation twinning modes [10]. Figure 
5 shows the model $\alpha-U$ grain structure, defined using DREAM.3D [50]. Based on pre-specified grain size and grain shape distributions this software generates a 3D synthetic, voxel-based structure and surface mesh for each individual grain. A 3D FE mesh is then built upon this microstructure for both grains and grain boundaries, ensuring mesh conformance between grain boundaries. The latter feature of the CPFE mesh is often refered to as 'conformal' grain boundaries.

Fig. 5. Twin formation and thickening with respect to the surrounding neighboring grains in the 3D model of $\alpha-U$ under compression in the Z direction with: (a) no twin, (b) $1 \%$ formed twin, (c) $3 \%$ twin, (d) one 5\% twin lamella, and (e) one of $2 \%$ and another of $3 \%$ volume fraction. The insert shows the distributions of normalized resolved shear stresses along the most dominant twin plane in the twin direction for the red interior grain [44].

The twin-free polycrystal in Fig. 5 was first compressed by imposing displacement in the normal direction, ND, with the lateral sides traction-free and, hence, free to expand. The stress state generated was consistent with that needed to form the (130)[310] twinning system in the central (red) grain. The twin lamella was then inserted in the red grain at the point of highest stress concentration in the grain boundary. As in the prior PBE and CPFFT studies described above, it was assumed that the twin boundaries were planar and spanned the entire grain crosssection. However in this case, the cross-sectional area and shape of the two flanking twin boundary planes were defined by the topology of the grain boundary they intersected, and their separation (thickness of the lamella), was calculated to produce a certain twin volume fraction. The twin lamella taking up $1 \%$ volume fraction of the central red grain is shown in Fig. $5 \mathrm{~b}$. Then, while the applied strain is held constant, the plastic deformation for all integration points in the twin lamella is enforced to correspond to the intrinsic twinning shear of a given twin variant, while plasticity for all integration points in the parent due to activity of the same variant is erased.

The CPFE discrete twinning model was used to study the effect of twinning on the stressstrain fields and relative activities of the slip families in $\alpha-U$. As found in the prior studies, when the twin lamella was introduced, the mechanical fields became highly heterogeneous, not only near the twin, but even far away from it within the parent. To expand the twin, an adjacent twin layer was added. In doing so, they found that at a critical twin thickness the stress fields within the twin changed. This observation led the authors to consider two possible pathways for the parent grain to achieve 5\% twin volume fraction: either by thickening the original twin from $3 \%$ to $5 \%$ or keeping the original $3 \%$ twin and forming a new lamella and growing it to $2 \%$. Between these two scenarios, the change in strain energy of the parent grain (as well as the polycrystal) was lower for the second one, suggesting that the formation of multiple twin lamellae would be energetically more favorable--qualitatively in agreement with the predictions for multiple twin formation in [40].

The three twin modeling methodologies reviewed above have demonstrated important progress in modeling discrete twin lamella in a microstructural framework. To reach this stage, many numerical obstacles have been overcome to introduce the characteristic twin reorientation and shear imposed within a finite, relatively thin domain of the twin. Much more progress is needed in order to model the evolution of deformation twins with strain, and ultimately calculate the constitutive response. First, we note that in all cases, the twins were inserted such that the twin boundaries were planar and the lamellae extended from one end of the grain to the other. 
This configuration represents a rather mature stage in twin formation and neglects the earlier stages of embryo creation and propagation of the twin. The pre-assigned twin shape of a planar lamella may also be too ideal. Twin boundaries of variable morphology have been observed in 2D and 3D [10, 12, 55-58]. Second, the dynamics of twin expansion have not been treated realistically. For instance, it has been implicitly assumed that twins expand layer by layer and homogeneously. Attempts have been made to capture some of these effects [37, 53, 54, 59], where twin nucleation and propagation was not chosen/pre-defined but instead controlled by a constitutive law. Geometries resulted in which the twin lamella did not necessarily extend completely across the parent grain, with propagation of twins instead dependent on local stress state. While this formulation certainly gave results in reasonable agreement with experimental observations, it highlighted a number of issues needing consideration. Most important of these are the criteria chosen for twin nucleation and propagation. While most models use a Schmid factor formulation (identical to slip) to determine when and which twins will form, it is recognized that a Schmid factor based on far-field stress is insufficient to describe variant selection [60]. The results from [54, 59] further suggest that the Schmid factor calculated based on local stress is insufficient to always correctly determine which twin variants form. While some authors [61] have suggested that it is the relative orientation of neighboring grain which is the deciding factor as to which twin variant will form, others have suggested [62] that it is local atomic scale mechanisms associated with dislocation nucleation and propagation that are key to determining which twin will form. Over the years, molecular dynamics simulation has provided a great of insight on the mechanisms underlying twin nucleation and growth [5]. Yet still, there is no widely accepted mechanism for twin nucleation and twin expansion. Continued progress in atomic-scale studies can provide the needed insight to develop physically based criteria for nucleating and propagating twins in the CPFE and FFT FE frameworks.

Nevertheless, despite these current drawbacks, the techniques discussed here are capable of studying local stress fields produced by twins. In this way, they can be used to potentially infer the likely processes that could occur later, such as void nucleation, double twinning, separate twin nucleation, twin-twin transmission across a boundary. Many of the key numerical barriers have been overcome making it relatively easier in the future to use these techniques to study phase transformations, other twin types in other crystal structures, growth twins, and shear bands.

\section{Acknowledgements}

This work is based upon project supported by the National Science Foundation under grant No. CMMI-1541918. IJB is grateful for the support from Los Alamos National Laboratory Directed Research and Development ER Grant 20140348ER.

\section{References}

[1] J.W. Christian, S. Mahajan, Progress in Materials Science, 39 (1995) 1-157.

[2] M.H. Yoo, Metall. Mater. Trans. A, 12 (1981) 409-418.

[3] S. Mendelson, Journal of Applied Physics, 41 (1970) 1893-1910.

[4] L. Capolungo, I. Beyerlein, Physical Review B, 78 (2008) 024117.

[5] I.J. Beyerlein, X. Zhang, A. Misra, Annual Review of Materials Research, 44 (2014) 329-

363.

[6] G.T. Gray III, Annual Review of Materials Research, 42 (2012) 285-303. 
[7] A.A. Salem, S.R. Kalidindi, R.D. Doherty, Acta Materialia, 51 (2003) 4225-4237.

[8] R.J. McCabe, G. Proust, E.K. Cerreta, A. Misra, International Journal of Plasticity, 25 (2009) 454-472.

[9] M. Knezevic, A. Levinson, R. Harris, R.K. Mishra, R.D. Doherty, S.R. Kalidindi, Acta Materialia, 58 (2010) 6230-6242.

[10] M. Knezevic, L. Capolungo, C.N. Tomé, R.A. Lebensohn, D.J. Alexander, B. Mihaila, R.J. McCabe, Acta Materialia, 60 (2012) 702-715.

[11] M. Knezevic, R.A. Lebensohn, O. Cazacu, B. Revil-Baudard, G. Proust, S.C. Vogel, M.E. Nixon, Materials Science and Engineering: A, 564 (2013) 116-126.

[12] M. Knezevic, M. Zecevic, I.J. Beyerlein, J.F. Bingert, R.J. McCabe, Acta Materialia, 88 (2015) 55-73.

[13] A. Akhtar, A. Teghtsoonian, Acta Metallurgica, 19 (1971) 655-663.

[14] B.S. Fromm, B.L. Adams, S. Ahmadi, M. Knezevic, Acta Materialia, 57 (2009) 2339-2348.

[15] F. Xu, R. Holt, M. Daymond, Acta Materialia, 56 (2008) 3672-3687.

[16] R.W. Cahn, Acta Crystallographica, 4 (1951) 470.

[17] M. Knezevic, R.J. McCabe, C.N. Tomé, R.A. Lebensohn, S.R. Chen, C.M. Cady, G.T. Gray Iii, B. Mihaila, International Journal of Plasticity, 43 (2013) 70-84.

[18] M. Knezevic, J. Crapps, I.J. Beyerlein, D.R. Coughlin, K.D. Clarke, R.J. McCabe, International Journal of Mechanical Sciences, 105 (2016) 227-238.

[19] M. Zecevic, M. Knezevic, I.J. Beyerlein, R.J. McCabe, Journal of Nuclear Materials, 473 (2016) 143-156.

[20] T.R. Bieler, L. Wang, A.J. Beaudoin, P. Kenesei, U. Lienert, Metallurgical and Materials Transactions A, 45 (2014) 109-122.

[21] B.M. Morrow, E.K. Cerreta, R.J. McCabe, C.N. Tomé, Materials Science and Engineering: A, 613 (2014) 365-371.

[22] B. Morrow, R. McCabe, E. Cerreta, C. Tomé, Metallurgical and Materials Transactions A, 45 (2014) 36-40.

[23] M. Zecevic, M. Knezevic, I.J. Beyerlein, C.N. Tomé, Materials Science and Engineering: A, 638 (2015) 262-274.

[24] M. Knezevic, I.J. Beyerlein, D.W. Brown, T.A. Sisneros, C.N. Tomé, International Journal of Plasticity, 49 (2013) 185-198.

[25] A. Levinson, R.K. Mishra, R.D. Doherty, S.R. Kalidindi, Acta Materialia, 61 (2013) 59665978.

[26] R.J. McCabe, A.W. Richards, D.R. Coughlin, K.D. Clarke, I.J. Beyerlein, M. Knezevic, Journal of Nuclear Materials, 465 (2015) 189-195.

[27] J. Koike, N. Fujiyama, D. Ando, Y. Sutou, Scripta Materialia, 63 (2010) 747-750.

[28] T. Al-Samman, G. Gottstein, Materials Science and Engineering: A, 488 (2008) 406-414.

[29] G.I. Taylor, Journal of the Institute of Metals, 62 (1938) 307-324.

[30] M. Knezevic, S.R. Kalidindi, D. Fullwood, International Journal of Plasticity, 24 (2008)

1264-1276.

[31] R.A. Lebensohn, C.N. Tomé, Acta Metallurgica et Materialia, 41 (1993) 2611-2624.

[32] P. Van Houtte, Acta Metallurgica et Materialia, 26 (1978) 591-604.

[33] C.N. Tomé, R.A. Lebensohn, U.F. Kocks, Acta Metallurgica et Materialia, 39 (1991) 26672680.

[34] X. Wu, S.R. Kalidindi, C. Necker, A.A. Salem, Acta Materialia, 55 (2007) 423-432.

[35] G. Proust, C.N. Tomé, G.C. Kaschner, Acta Materialia, 55 (2007) 2137-2148. 
[36] C. Mareau, M.R. Daymond, Acta Materialia, 58 (2010) 3313-3325.

[37] H. Abdolvand, M. Majkut, J. Oddershede, J.P. Wright, M.R. Daymond, Acta Materialia, 93 (2015) 246-255.

[38] C. Aydıner, J. Bernier, B. Clausen, U. Lienert, C. Tomé, D. Brown, Physical Review B, 80 (2009) 024113.

[39] H. Abdolvand, M. Majkut, J. Oddershede, S. Schmidt, U. Lienert, B.J. Diak, P.J. Withers, M.R. Daymond, International Journal of Plasticity, 70 (2015) 77-97.

[40] L. Balogh, S. Niezgoda, A. Kanjarla, D. Brown, B. Clausen, W. Liu, C. Tomé, Acta Materialia, 61 (2013) 3612-3620.

[41] R.Y. Zhang, M.R. Daymond, R.A. Holt, Materials Science and Engineering: A, 473 (2008) 139-146.

[42] M.R. Barnett, N. Stanford, A. Ghaderi, F. Siska, Acta Materialia, 61 (2013) 7859-7867. [43] M. Arul Kumar, A.K. Kanjarla, S.R. Niezgoda, R.A. Lebensohn, C.N. Tomé, Acta Materialia, 84 (2015) 349-358.

[44] M. Ardeljan, R.J. McCabe, I.J. Beyerlein, M. Knezevic, Computer Methods in Applied Mechanics and Engineering, 295 (2015) 396-413.

[45] M. Knezevic, R.J. McCabe, R.A. Lebensohn, C.N. Tomé, C. Liu, M.L. Lovato, B. Mihaila, Journal of the Mechanics and Physics of Solids, 61 (2013) 2034-2046.

[46] M. Zecevic, R.J. McCabe, M. Knezevic, Mechanics of Materials, 84 (2015) 114-126.

[47] S.R. Kalidindi, H.K. Duvvuru, M. Knezevic, Acta Materialia, 54 (2006) 1795-1804.

[48] S.R. Kalidindi, C.A. Bronkhorst, L. Anand, Journal of the Mechanics and Physics of Solids, 40 (1992) 537-569.

[49] M. Ardeljan, M. Knezevic, T. Nizolek, I.J. Beyerlein, N.A. Mara, T.M. Pollock, International Journal of Plasticity, 74 (2015) 35-57.

[50] DREAM.3D Version 4.2, BlueQuartz Software, Springboro, OH, USA, (2013).

[51] D.J. Savage, M. Knezevic, Computational Mechanics 56 (2015) 677-690.

[52] R.Y. Zhang, M.R. Daymond, R.A. Holt, Materials Science and Engineering: A, 528 (2011) 2725-2735.

[53] H. Abdolvand, M.R. Daymond, C. Mareau, International Journal of Plasticity, 27 (2011) 1721-1738.

[54] H. Abdolvand, M.R. Daymond, Journal of the Mechanics and Physics of Solids, 61 (2013) 803-818.

[55] N. Zaafarani, D. Raabe, R. Singh, F. Roters, S. Zaefferer, Acta Materialia, 54 (2006) 18631876.

[56] S. Yi, I. Schestakow, S. Zaefferer, Materials Science and Engineering: A, 516 (2009) 58-64. [57] J. Lind, S.F. Li, R. Pokharel, U. Lienert, A.D. Rollett, R.M. Suter, Acta Materialia, 76 (2014) 213-220.

[58] C.A. Stein, A. Cerrone, T. Ozturk, S. Lee, P. Kenesei, H. Tucker, R. Pokharel, J. Lind, C. Hefferan, R.M. Suter, A.R. Ingraffea, A.D. Rollett, Current Opinion in Solid State and Materials Science, 18 (2014) 244-252.

[59] H. Abdolvand, M. Majkut, J. Oddershede, J.P. Wright, M.R. Daymond, Acta Materialia, 93 (2015) 235-245.

[60] L. Capolungo, P. Marshall, R. McCabe, I. Beyerlein, C. Tomé, Acta Materialia, 57 (2009) 6047-6056.

[61] J.J. Jonas, S. Mu, T. Al-Samman, G. Gottstein, L. Jiang, Ė. Martin, Acta Materialia, 59 (2011) 2046-2056. 
[62] I. Beyerlein, L. Capolungo, P. Marshall, R. McCabe, C. Tomé, Philosophical Magazine, 90 (2010) 2161-2190. 

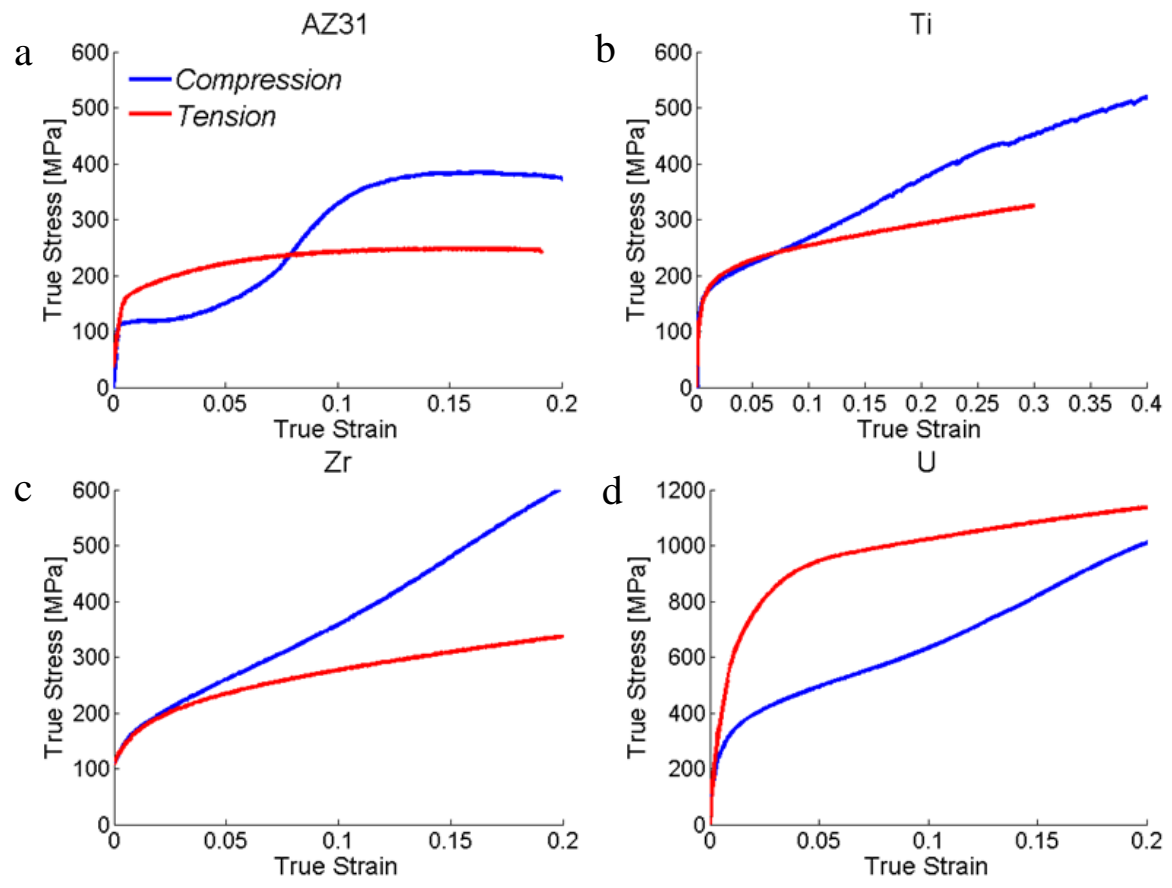

Fig. 1. Effect of deformation twinning on the compressive response of: (a) AZ31 [9], (b) $\alpha$-Ti [11], (c) $\alpha-Z r$ [12], and (d) $\alpha-U$ [10]. Response in tension for all these metals exhibits characteristics of slip dominated deformation. 

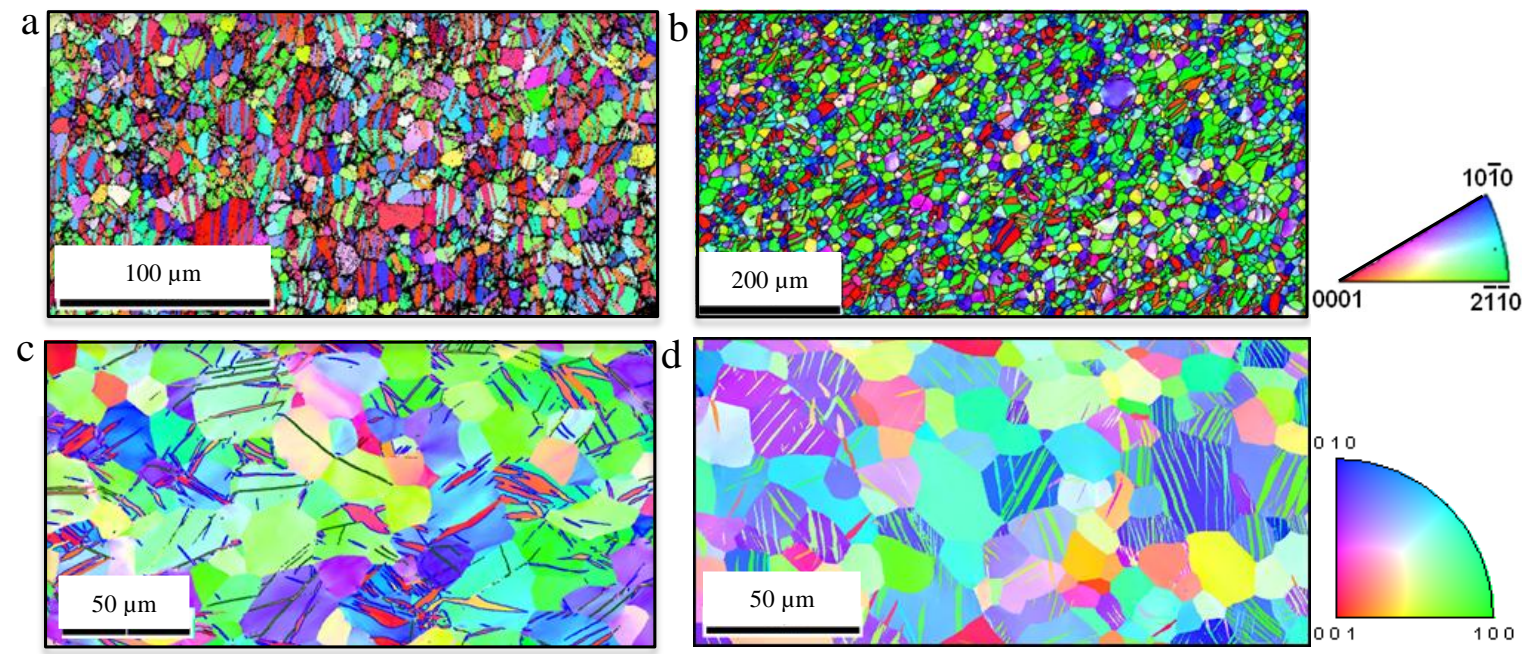

Fig. 2. Inverse pole figure maps showing microstructure evolution in samples compressed along the in-plane rolling direction of sheets of (a) AZ31 to a true strain of 0.04 at $298 \mathrm{~K}$ [9], (b) $\alpha$-Ti to a true strain of 0.1 at $298 \mathrm{~K}$ [11], (c) $\alpha-\mathrm{Zr}$ to a true strain of 0.08 at $76 \mathrm{~K}$ [12], and (d) $\alpha-\mathrm{U}$ to a true strain of 0.05 at $298 \mathrm{~K}$ [10]. These maps indicate the orientation of the compression axis with respect to the crystal reference frame. 
a
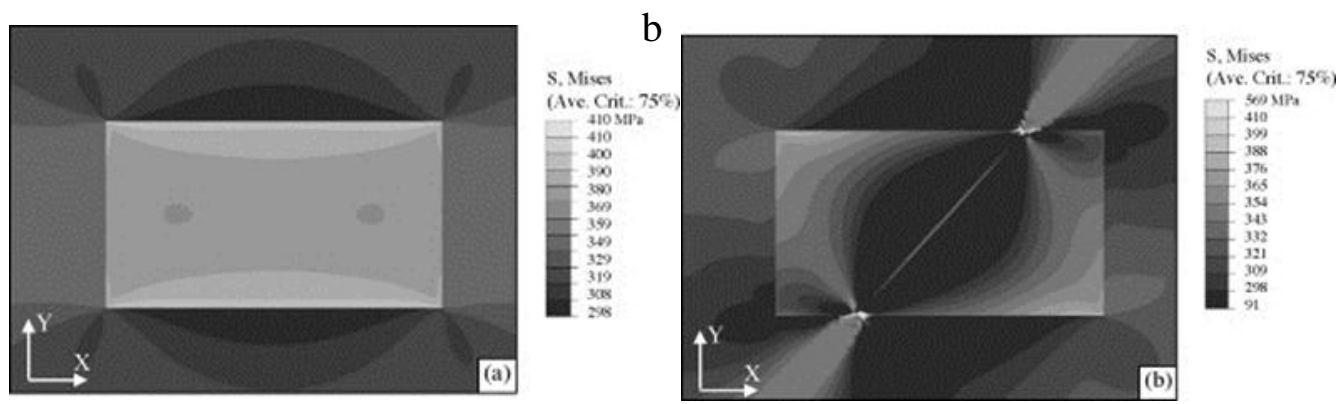

Fig. 3. A 2D FE model for twinning with a square inclusion represents a grain embedded in an isotropic surrounding medium. Von Mises stress contour before (a) and after (b) twinning under tension along the global $\mathrm{X}$ direction [41]. 


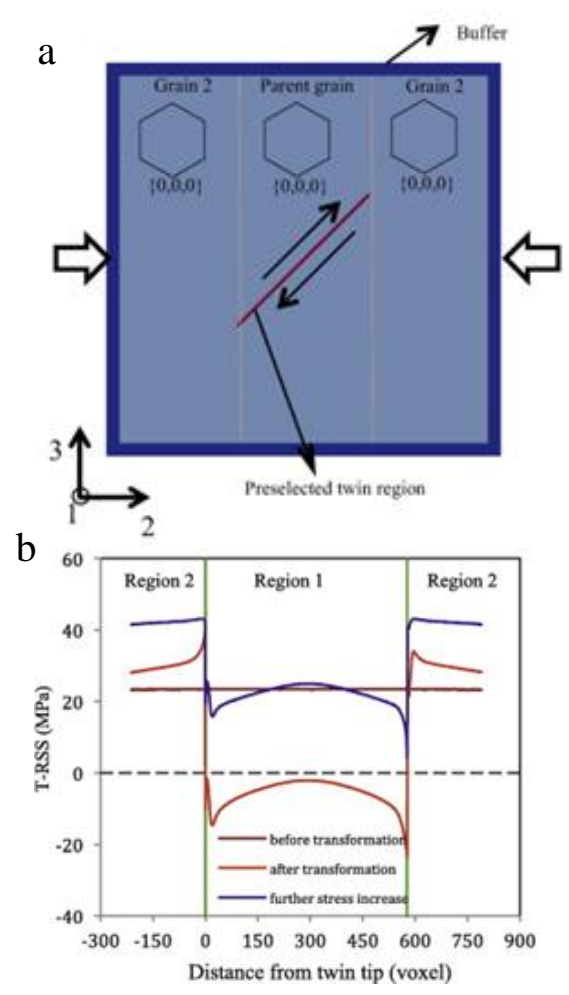

Fig. 4. (a) Schematic of the tricrystal unit cell compressed in the 2-direction with a twin inside the central crystal and (b) the resolved shear stress profile along the twin-parent grain boundary [43]. 

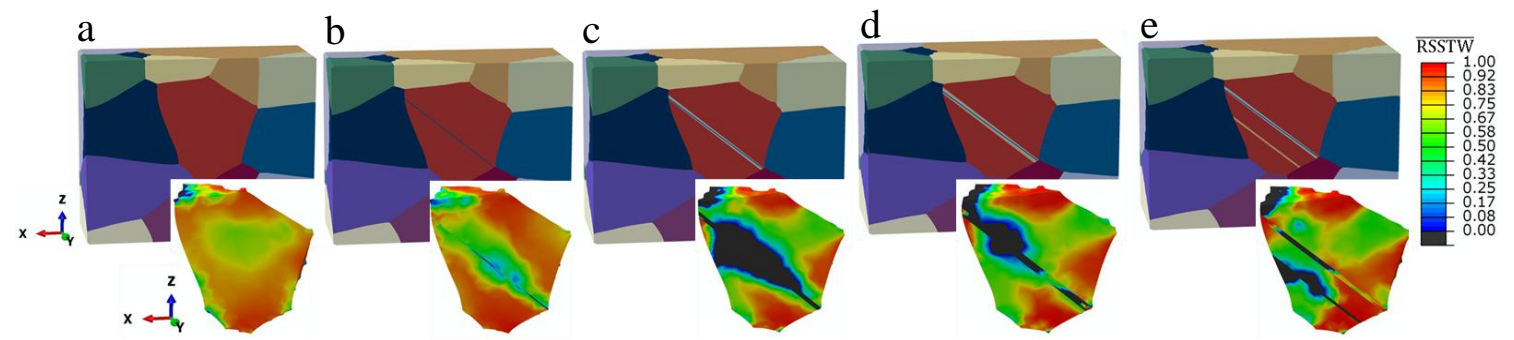

Fig. 5. Twin formation and thickening with respect to the surrounding neighboring grains in the 3D model of $\alpha-U$ under compression in the Z direction with: (a) no twin, (b) $1 \%$ formed twin, (c) $3 \%$ twin, (d) one 5\% twin lamella, and (e) one of $2 \%$ and another of $3 \%$ volume fraction. The insert shows the distributions of normalized resolved shear stresses along the most dominant twin plane in the twin direction for the red interior grain [44]. 